\title{
1 Screw insertion in trabecular bone causes peri-implant bone damage
}

2

3 Juri A. Steiner ${ }^{1}$, Stephen J. Ferguson ${ }^{1}$, G. Harry van Lenthe ${ }^{1,2}$

4

$5 \quad{ }^{1}$ Institute for Biomechanics, ETH Zurich, Vladimir-Prelog-Weg 3, 8093 Zurich, Switzerland

6 '2Biomechanics Section, KU Leuven - University of Leuven, Celestijnenlaan 300, 3001

7 Leuven, Belgium

8

9

10 Corresponding author:

11 Juri A. Steiner

12 Institute for Biomechanics, ETH Zurich,

13 HPP O 14 , Hönggerbergring 64, 8093 Zurich, Switzerland

14 Phone: +41446334013

15 Email: steineju@ethz.ch

16

17

Word count: 2'981 


\section{Abstract}

2 Secure fracture fixation is still a major challenge in orthopedic surgery, especially in osteoporotic bone. While numerous studies have investigated the effect of implant loading on the peri-implant bone after screw insertion, less focus has been put on bone damage that may occur due to the screw insertion process itself. Therefore, the aim of this study was to localize and quantify peri-implant bone damage caused by screw insertion.

We used non-invasive three-dimensional micro-computed tomography to scan twenty human femoral bone cores before and after screw insertion. After image registration of the pre- and post-insertion scans, changes in the bone micro-architecture were identified and quantified. This procedure was performed for screws with a small thread size of $0.3 \mathrm{~mm}$ (STS, $\mathrm{N}=10)$ and large thread size of $0.6 \mathrm{~mm}(\mathrm{LTS}, \mathrm{N}=10)$.

Most bone damage occurred within a $0.3 \mathrm{~mm}$ radial distance of the screws. Further bone damage was observed up to $0.6 \mathrm{~mm}$ and $0.9 \mathrm{~mm}$ radial distance from the screw, for the STS and LTS groups, respectively. While a similar amount of bone damage was found within a $0.3 \mathrm{~mm}$ radial distance for the two screw groups, there was significantly more bone damage for the LTS group than the STS group in volumes of interest between 0.3-0.6 mm and 0.6-0.9 mm.

In conclusion, this is the first study to localize and quantify peri-implant bone damage caused by screw insertion based on a non-invasive, three-dimensional, micro-CT imaging technique. We demonstrated that peri-implant bone damage already occurs during screw insertion. This should be taken into consideration to further improve primary implant stability, especially in low quality osteoporotic bone. We believe that this technique could be a promising method to assess more systematically the effect of peri-implant bone damage on primary implant stability. Furthermore, including peri-implant bone damage due to screw insertion into patient-specific in silico models of implant-bone systems could improve the accuracy of these models. 


\section{INTRODUCTION}

Secure fixation of bone fragments after a fracture is mandatory to achieve proper healing. Consequently, substantial efforts have gone towards improving fracture fixation. It has been demonstrated that implant design, implant surface characteristics [1], [2] and surgical technique [3]-[5] can all considerably influence the clinical outcome.

Not only the implant, but also the bone itself plays a role in achieving proper fixation. Secure fracture fixation is still a major challenge, especially in osteoporotic bone. First, primary stability is decreased because screws have less purchase in osteoporotic bone [6][8]; second, secondary stability is negatively affected because osteoporotic bone shows a diminished healing capacity [9]-[12]. As the elderly population increases and continues to age, the number of osteoporotic fracture incidents is increasing [13], [14]. These biomechanical and demographic changes stress the importance to further improve fracture fixation in osteoporotic bone.

While the effect of implant loading on the peri-implant bone has been investigated after screw insertion [5], [15]-[20], less focus has been put on the bone damage caused by screw insertion itself [1], [21]-[24]. At the micro-scale, different modes of micro-damage (e.g. micro-cracks, cross hatch damage and diffuse damage) have been localized and quantified using fluorescent microscopy based histomorphometry [1], [22], [24]and/or laser scanning microscopy [21]. However, none of these methods have been able to capture and/or quantify peri-implant damage fully in three dimensions (3D). Furthermore, histomorphometry is invasive per se and, consequently, does not allow for a direct comparison between the 'before' and 'after' screw insertion state within the same specimen. Moreover, it is not clear to what extent the preparation of the histomorphometric slices creates micro-damage in the bone. Therefore, the aim of this study was to capture and analyze bone damage due to screw insertion and to do so non-invasively and fully in three dimensions. We hypothesized that bone damage would occur close to the implant only, and that it would be affected by the screw thread design. 


\section{METHODS}

Twenty cylindrical trabecular bone specimens (18 $\mathrm{mm}$ height; $16 \mathrm{~mm}$ diameter) were core drilled from the epiphyseal region of twelve cadaveric human femoral heads (age $66.5 \pm 10.5$ years). After predrilling with a diameter of $2 \mathrm{~mm}$, one self-tapping screw (length: 16mm; core diameter: $2.1 \mathrm{~mm}$ ) was inserted $11 \mathrm{~mm}$ into in the center of each specimen according to the manufacturer's guidelines. Two thread sizes, defined as $0.5 \times$ (outer diameter - core diameter), were used. The small thread size (STS) and large thread size (LTS) were $0.3 \mathrm{~mm}$ and $0.6 \mathrm{~mm}$, respectively. The pitch sizes of the STS and LTS groups were 0.65 and 1.31 , respectively. All specimens were scanned twice with micro-computed tomography $(\mu \mathrm{CT} 50$, Scanco Medical, Brüttisellen, Switzerland) using $90 \mathrm{kVp}$ energy at a nominal isotropic resolution of $20 \mu \mathrm{m}$; one scan was made of the intact specimen prior to screw insertion and another scan was made just after screw insertion. The screws were custom-made (DePuy Synthes, Solothurn) based on a Titanium-Aluminum alloy $\left(\mathrm{TiAl}_{6} \mathrm{Nb}_{7}\right)$ that contains a relatively low atomic number to reduce potential metal artefacts during scanning [25]. After image reconstruction, rigid image registration was performed, as described in recently published literature [26], to align the two images (Fig. 1a and 1b). Next, both images were segmented following well accepted guidelines [27] (Fig. 1c) based on visually distinctive grey scale values for bone and screw (arbitrary units: 200 to 400 and 800 and 1000, respectively). A visual check revealed no artificial gaps due to the segmentation. Next, the segmented screw from the physical insertion (PI) model was inserted digitally (Fig. 1d) into the image stack of the pre-insertion scan to obtain a digital insertion (DI) model. While the PI model represents the bone core with the physically inserted screw including the peri-implant bone damage (Fig. 2a), the DI model represents an idealized perfect insertion without any peri-implant bone damage (Fig. 2b). Bone debris at the outer surface of the bone sample, caused by sample preparation, was extracted by digitally removing a $0.5 \mathrm{~mm}$ ring of the outermost bone structure. Next, an image overlay was performed between the PI screw model and the DI screw model (Fig. 3). In the overlay image, two specific voxel-labeling categories were 
1 defined: (1) bone present in both images (transparent grey in Fig. 3) and (2) bone present in

2 the PI image only (pink in Fig. 3). The bone in category 2 represents bone that has been

3 displaced due to pre-hole drilling and screw insertion and is quantified as the bone damage volume (BDV; Fig. 3). Bone damage density (BDD) was assessed by computing BDV normalized to the bone volume $(\mathrm{BV})$ in hollow cylindrical VOls around the screw (BDD= BDV/BV) of the corresponding cylindrical VOI. All VOls had a radial thickness of $0.3 \mathrm{~mm}$; their inner radius was varied in steps of $0.3 \mathrm{~mm}$ (up to $5.1 \mathrm{~mm}$ ) to describe different distances to the screw (Fig. 4a). BV divided by the total volume (TV) in which the BV is located, was computed to calculate the average bone volume fraction (BV/TV) over all specimens as previously defined here [27]. Furthermore, BV/TV was assessed within each hollow cylindrical VOI and correlated to BDD.

Most measurement techniques contain a residual noise level (i.e. precision) even though in reality no signal exists. In our case, the precision of the entire rescanning and registration process was assessed underneath the screw where no architectural changes were expected. More specifically, all bone voxels belonging to category 2 were quantified in 14 cylindrical volumes of interest (VOI; all $0.3 \mathrm{~mm}$ in height and $5.4 \mathrm{~mm}$ in diameter) underneath the screws and normalized to BV of the VOI (Fig. 4b). The quantified precision from the region underneath the screw (Fig. 4b) served as threshold against which BDD values in radial distance to the screw (Fig. 4a) were compared to detect actual bone damage that is significantly different from the precision level.

\section{Statistical analyses}

ANOVA multi-comparison test with Bonferroni correction was used to determine whether BDD differed significantly $(\alpha=0.05)$ from the precision level. 


\section{RESULTS}

No significant differences in precision were found between any of the VOls underneath the screws of the STS and LTS group. Hence, the data were pooled and served as the precision level against which all VOls in the radial direction to the screws could be compared. For the STS and LTS group, the precision was $0.072 \pm 0.02 \%$ and $0.072 \pm 0.03 \%$, respectively.

A visual comparison between the PI and DI model showed clear evidence for peri-implant bone damage due to screw insertion (indicated by red arrows in Fig. 2). Further visual assessment of the computed bone damage revealed a clear densification of BDD towards the implant site (Fig. 5a and 5b). For the STS group, BDD was significantly higher than the precision up to $0.6 \mathrm{~mm}$ distance from the screw (Fig. 5c). For the LTS group significant differences were found up to $0.9 \mathrm{~mm}$ radial distance from the implant (Fig. 5d). In both groups, we could observe that with increasing distance from the screw, BDD quickly converged towards the precision level (Fig. $5 c$ and $5 d$ ). The LTS group showed significantly higher BDD values than the STS group in the VOls between 0.3-0.6 and 0.6-0.9 mm distance from the implant. Highest BDD values were found in the VOI closest to the implant; no significant differences in BDD were found between the STS and LTS groups. The average BV/TV over all specimens is $27.2 \% \pm 4.1 \%$. BV/TV for the STS and LTS group was $25.8 \pm 1.9 \%$ and $29.0 \pm 4.6 \%$, respectively $(p=0.064)$. Within the VOls where BDD was significantly higher than the precision, BV/TV shows no significant correlation to BDD in both groups $\left(R^{2}<0.4\right.$ and $\left.p>0.05\right)$.

\section{DISCUSSION}

The aim of this study was to develop a non-invasive method that would allow the qualitative and quantitative assessment of peri-implant bone damage caused by screw insertion. This goal was achieved. We could show that the trabecular bone structure is affected by screw insertion. Bone damage was found close to the implant only. We also showed that screws with large threads had a slightly larger impact on the peri-implant bone than screws with 
small threads. Bone damage does not seem to depend on bone volume fraction. Low correlation coefficients were found for other morphometric parameters, too. We assume that the accumulation of peri-implant bone damage is a complex phenomenon that depends on the underlying composition of the bone tissue. For instance, the ultrastructure of the bone (i.e. collagen content and its orientation) is known to have an influence on the mechanical properties (e.g. brittleness) of bone tissue and might also play an important role on the fracture mechanisms during screw insertion, hence on the amount of peri-implant damage. However, collagen cannot be assessed using microCT. Hence, for future studies, it would be interesting to use more sophisticated measurement techniques (e.g. 3D scanning SAXS [28]) to investigate the complex relationship between bone quality and peri-implant bone damage caused by screw insertion. Unfortunately, 3D scanning SAXS is an invasive technique and only a very limited number of individual trabeculae can be assessed per specimen due the complex methodology that requires vast computational resources. Further improvements in the measurement technique and computational performance are required to make this method applicable in a non-invasive fashion for larger volumes of interest as well as larger samples sizes. The impaction of the bone during screw insertion can increase the implant-bone stability [29]. During this process, bony pieces can break off from the trabecular network and can serve as void filler which might enhance primary implant stability. An even higher stability would be excepted if a perfectly intact peri-implant bone could be preserved during screw insertion. However, this is not the case. We recently conducted a computational study in which we found that despite the increased bone-screw interface surface of the bone-screw composite, its mechanical competence is decreased compared to models in which screws were placed digitally with perfectly intact peri-implant bone [30]. State-of-the-art computational models have demonstrated high over-predictions of the primary implant stability in screw-bone systems [31]-[33]. This is probably due to the fact that perfectly intact bone-screw interfaces were assumed. Hence, even though bone compaction can enhance primary implant stability in some cases [29], there is clear 
1 evidence, that local peri-implant bone damage occurs and hampers the mechanical

2 performance of bone-implant systems when compared to idealized implant placement with

3 perfectly preserved and intact peri-implant bone.

4 LTS screws induced more peri-implant damage than STS screws did. Based on these

5 data we can conclude that the larger the outer diameter of a screw the more damage is

6 induced in the peri-implant bone. However, corresponding in vitro mechanical tests

7 (data not shown here) indicate no significant difference in bone-implant stiffness

8 between STS and LTS screws. Furthermore, as osseointegration is known to be

9 initiated by lesions of the pre-existing bone matrix [34], increased BDD could potentially

10 correlate with improved secondary implant stability. Hence, further research is required

11 to investigate the effect of BDD on the mechanical competence on primary and secondary implant stability. For this purpose, in vivo longitudinal studies in animals would be required as described here [35]. 
1 present study is that a non-invasive micro-CT imaging technique was used with which the

2 bone damage around the entire screw was visualized and quantified.

State-of-the-art patient-specific computational analysisare not able to accurately

simulate the mechanical competence of orthopedic screws placed into human bone. Hence, the inclusion of a peri-implant bone damage region with reduced mechanical competence could potentially improve the accuracy of such in silico computational models. The knowledge gained from this study could be useful to improve the accuracy of patient-specific high-resolution micro-CT based finite element ( $\mu \mathrm{FE})$ analysis of implant-bone systems. While patient-specific finite element (FE) computer simulations of trabecular bone alone have shown to be in very good agreement with corresponding in vitro mechanical test [40][42], these in silico models tend to overestimate the mechanical response in implant-bone systems [31], [43].

This study has several limitations: First, metal screws were used that create metal artifacts during micro-CT scanning. However, specific measures, such as high energy scans and titanium-aluminum alloy based screws were used to reduce the metal artifact. Second, the current setup does not allow distinguishing the bone damage caused by the drilling procedure from the bone damage caused by screw insertion, as only two sets of scans per specimen were conducted (i.e. before guide hole drilling and after screw insertion). Nevertheless, the comparison of the two different screw types is valid because the exact same guide hole drilling procedure was used in both groups. Third, the method identifies only fragmentation of peri-implant bone trabeculae, not micro-damage within the trabeculae themselves. The current image resolution of $20 \mu \mathrm{m}$ is not sufficient to assess peri-implant bone damage at the micro-scale (e.g. micro-cracks, cross-hatched damage or diffuse damage). This is due to the fact that micro-damage occurs at a length-scale that is at least one order of magnitude smaller. Fourth, depending on the trabecular dimensions and possible residual stresses in the trabecular network, elastic bending of trabeculae may be interpreted as damage by this registration method. However, structural changes captured at 
$120 \mu \mathrm{m}$ can most likely be interpreted as bone damage considering the fact the micro-cracks 2 already occur at dimensions of 1 to $2 \mu \mathrm{m}$.

3

In conclusion, this study is the first to localize and quantify peri-implant bone damage along the entire length of the screw; furthermore, this was done non-invasively and fully in $3 \mathrm{D}$. We believe that this technique could be a promising method to investigate more systematically the effect of peri-implant bone damage on primary implant stability, such as caused by excessive loading and screw insertion. Furthermore, including peri-implant bone damage due to screw insertion into patient-specific in silico models of implant-bone systems could improve the accuracy of these models. 
1 Funding:

2 This study was funded in part by the Swiss Commission for Technology and Innovation (CTI)

3 through grant KTI-Nr. 14067.1 PFLS-LS and by Synthes $\mathrm{GmbH}$.

4

5 Conflicts of Interest:

6 None declared

7

8 Ethical Approval:

9 Bone cores were obtained from resected bones from orthopedic surgical interventions in 10 collaboration with Schulthess Clinic (Zurich, Switzerland). The corresponding ethics 11 committee approval (EK-29/2007) allows measurements by $\mu \mathrm{CT}$ as well as additional 12 experimental experiments. 


\section{$\underline{\text { Reference List }}$}

[1] P. M. Bartold, J. S. Kuliwaba, V. Lee, S. Shah, V. Marino, and N. L. Fazzalari, "Influence of surface roughness and shape on microdamage of the osseous surface adjacent to titanium dental implants.," Clin. Oral Implants Res., vol. 22, no. 6, pp. 613-8, Jun. 2011.

[2] M. V. Dos Santos, C. N. Elias, and J. H. Cavalcanti Lima, "The effects of superficial roughness and design on the primary stability of dental implants.," Clin. Implant Dent. Relat. Res., vol. 13, no. 3, pp. 215-23, Sep. 2011.

[3] M. I. Fanuscu, T.-L. Chang, and K. Akça, "Effect of surgical techniques on primary implant stability and peri-implant bone.," J. Oral Maxillofac. Surg., vol. 65, no. 12, pp. 2487-91, Dec. 2007.

[4] F. Javed, H. B. Ahmed, R. Crespi, and G. E. Romanos, "Role of primary stability for successful osseointegration of dental implants: Factors of influence and evaluation.," Interv. Med. Appl. Sci., vol. 5, no. 4, pp. 162-7, Dec. 2013.

[5] A. Büchter, J. Kleinheinz, H. P. Wiesmann, J. Kersken, M. Nienkemper, H. Von Weyhrother, U. Joos, and U. Meyer, "Biological and biomechanical evaluation of bone remodelling and implant stability after using an osteotome technique.," Clin. Oral Implants Res., vol. 16, no. 1, pp. 1-8, Feb. 2005.

[6] F. Bonnaire, H. Zenker, C. Lill, A. T. Weber, and B. Linke, "Treatment strategies for proximal femur fractures in osteoporotic patients.," Osteoporos. Int., vol. 16 Suppl 2, pp. S93-S102, Mar. 2005.

[7] J. Seebeck, J. Goldhahn, H. Städele, P. Messmer, M. M. Morlock, and E. Schneider, "Effect of cortical thickness and cancellous bone density on the holding strength of internal fixator screws.," J. Orthop. Res., vol. 22, no. 6, pp. 1237-42, Nov. 2004.

[8] O. C. Thiele, C. Eckhardt, B. Linke, E. Schneider, and C. a Lill, "Factors affecting the stability of screws in human cortical osteoporotic bone: a cadaver study.," J. Bone Joint Surg. Br., vol. 89, no. 5, pp. 701-5, May 2007.

[9] C. Lill and J. Hesseln, "Biomechanical evaluation of healing in a non-critical defect in a large animal model of osteoporosis," J. Orthop. Res., vol. 21, pp. 836-842, 2003.

[10] R. Bindl, R. Oheim, P. Pogoda, F. T. Beil, K. Gruchenberg, S. Reitmaier, T. Wehner, E. Calcia, P. Radermacher, L. Claes, M. Amling, and A. Ignatius, "Metaphyseal fracture healing in a sheep model of low turnover osteoporosis induced by hypothalamic-pituitary disconnection (HPD).," J. Orthop. Res., vol. 31, no. 11, pp. 1851-7, Nov. 2013.

[11] T. Kubo, T. Shiga, J. Hashimoto, M. Yoshioka, H. Honjo, M. Urabe, I. Kitajima, I. Semba, and Y. Hirasawa, "Osteoporosis influences the late period of fracture healing in a rat model prepared by ovariectomy and low calcium diet.," J. Steroid Biochem. Mol. Biol., vol. 68, no. 5-6, pp. 197-202, Mar. 1999. 
influence of osteoporosis in femoral fracture healing time.," Injury, vol. 40, no. 6, pp. 663-8, Jun. 2009.

[13] J. A. Kanis, O. Johnell, A. Oden, C. De Laet, and D. Mellstrom, "Epidemiology of osteoporosis and fracture in men.," Calcif. Tissue Int., vol. 75, no. 2, pp. 90-9, Aug. 2004.

[14] O. Johnell and J. A. Kanis, "An estimate of the worldwide prevalence and disability associated with osteoporotic fractures.," Osteoporos. Int., vol. 17, no. 12, pp. 1726-33, Dec. 2006.

[15] S. S. Huja, T. R. Katona, D. B. Burr, L. P. Garetto, and W. E. Roberts, "Microdamage adjacent to endosseous implants," Bone, vol. 25, no. 2, pp. 217-222, Aug. 1999.

[16] S. S. Huja, M. Sayeed Hasan, R. Pidaparti, C. H. Turner, L. P. Garetto, and D. B. Burr, "Development of a fluorescent light technique for evaluating microdamage in bone subjected to fatigue loading," J. Biomech., vol. 32, no. 11, pp. 1243-1249, Nov. 1998.

[17] S. E. Basler, J. Traxler, R. Müller, and G. H. van Lenthe, "Peri-implant bone microstructure determines dynamic implant cut-out.," Med. Eng. Phys., vol. 35, no. 10, pp. 1442-9, Oct. 2013.

[18] T. L. Mueller, S. E. Basler, R. Müller, and G. H. van Lenthe, "Time-lapsed imaging of implant fixation failure in human femoral heads.," Med. Eng. Phys., vol. 35, no. 5, pp. 636-43, May 2013.

[19] J. Duyck, K. Vandamme, L. Geris, H. Van Oosterwyck, M. De Cooman, J. Vander Sloten, R. Puers, and I. Naert, "The influence of micro-motion on the tissue differentiation around immediately loaded cylindrical turned titanium implants.," Arch. Oral Biol., vol. 51, no. 1, pp. 1-9, Jan. 2006.

[20] F. Isidor, "Influence of forces on peri-implant bone," Clin. Oral Implants Res., pp. 8-18, 2006.

[21] L. Wang, J. Shao, T. Ye, L. Deng, and S. Qiu, "Three-dimensional morphology of microdamage in peri-screw bone: a scanning electron microscopy of methylmethacrylate cast replica.," Microsc. Microanal., vol. 18, no. 5, pp. 1106-11, Oct. 2012.

[22] A. Warreth, I. Polyzois, C. T. Lee, and N. Claffey, "Generation of microdamage around endosseous implants.," Clin. Oral Implants Res., vol. 20, no. 12, pp. 1300-6, Dec. 2009.

[23] C. Wawrzinek, T. Sommer, and H. Fischer-Brandies, "Microdamage in cortical bone due to the overtightening of orthodontic microscrews.," J. Orofac. Orthop., vol. 69, no. 2, pp. 121-34, Mar. 2008.

[24] E. M. Clary and S. C. Roe, "In vitro biomechanical and histological assessment of pilot hole diameter for positive-profile external skeletal fixation pins in canine tibiae.," Vet. Surg., vol. 25, no. 6, pp. 453-62, 1996.

[25] C. Zannoni, M. Viceconti, L. Pierotti, and A. Cappello, "Analysis of titanium induced CT artifacts in the development of biomechanical finite element models," Med. Eng. Phys., vol. 20, no. 9, pp. 653-659, Dec. 1998. 
[26] F. a Schulte, F. M. Lambers, D. J. Webster, G. Kuhn, and R. Müller, "In vivo validation of a computational bone adaptation model using open-loop control and time-lapsed micro-computed tomography.," Bone, vol. 49, no. 6, pp. 1166-72, Dec. 2011.

[27] M. L. Bouxsein, S. K. Boyd, B. A. Christiansen, R. E. Guldberg, K. J. Jepsen, and R. Müller, "Guidelines for assessment of bone microstructure in rodents using micro-computed tomography," J. Bone Miner. Res., vol. 25, no. 7, pp. 1468-1486, 2010.

[28] M. Georgiadis, M. Guizar-Sicairos, A. Zwahlen, A. J. Trüssel, O. Bunk, R. Müller, and P. Schneider, "3D scanning SAXS: A novel method for the assessment of bone ultrastructure orientation," Bone, vol. 71, pp. 42-52, 2015.

[29] T. Shea and J. Laun, "Designs and Techniques That Improve the Pullout Strength of Pedicle Screws in Osteoporotic Vertebrae: Current Status," BioMed Res. ..., vol. 2014, 2014.

[30] A. J. Steiner, S. J. Ferguson, and G. H. van Lenthe, "Screw insertion has a negative impact on peri-implant bone quality," 5th Int. Conf. Comput. Bioeng., 2013.

[31] A. J. Wirth, T. L. Mueller, W. Vereecken, C. Flaig, P. Arbenz, R. Müller, and G. H. Lenthe, "Mechanical competence of bone-implant systems can accurately be determined by imagebased micro-finite element analyses," Arch. Appl. Mech., vol. 80, no. 5, pp. 513-525, Oct. 2009.

[32] A. Torcasio, X. Zhang, H. Van Oosterwyck, J. Duyck, and G. H. van Lenthe, "Use of micro-CTbased finite element analysis to accurately quantify peri-implant bone strains: a validation in rat tibiae.," Biomech. Model. Mechanobiol., vol. 11, no. 5, pp. 743-50, May 2012.

[33] K. Karunratanakul, G. Kerckhofs, J. Lammens, J. Vanlauwe, J. Schrooten, and H. Van Oosterwyck, "Validation of a finite element model of a unilateral external fixator in a rabbit tibia defect model.," Med. Eng. Phys., vol. 35, no. 7, pp. 1037-43, Jul. 2013.

[34] R. K. Schenk and D. Buser, "Osseointegration: a reality," Periodontol. 2000, vol. 17, no. 1, pp. 22-35, Jun. 1998.

[35] V. a Stadelmann, C. M. Conway, and S. K. Boyd, "In vivo monitoring of bone-implant bond strength by microCT and finite element modelling.," Comput. Methods Biomech. Biomed. Engin., vol. 16, no. 9, pp. 993-1001, Jan. 2013.

[36] P. J. Thurner, P. Wyss, R. Voide, M. Stauber, M. Stampanoni, U. Sennhauser, and R. Müller, "Time-lapsed investigation of three-dimensional failure and damage accumulation in trabecular bone using synchrotron light.," Bone, vol. 39, no. 2, pp. 289-99, Aug. 2006.

[37] A. Larrue, A. Rattner, Z.-A. Peter, C. Olivier, N. Laroche, L. Vico, and F. Peyrin, "Synchrotron radiation micro-CT at the micrometer scale for the analysis of the three-dimensional morphology of microcracks in human trabecular bone.," PLoS One, vol. 6, no. 7, p. e21297, Jan. 2011.

[38] X. Wang, D. B. Masse, H. Leng, K. P. Hess, R. D. Ross, R. K. Roeder, and G. L. Niebur, "Detection of trabecular bone microdamage by micro-computed tomography.," J. Biomech., vol. 40, no. 15, pp. 3397-403, Jan. 2007. 
[39] S. Y. Tang and D. Vashishth, "A non-invasive in vitro technique for the three-dimensional quantification of microdamage in trabecular bone.," Bone, vol. 40, no. 5, pp. 1259-64, May 2007.

[40] Y. Chevalier, D. Pahr, H. Allmer, M. Charlebois, and P. Zysset, "Validation of a voxel-based FE method for prediction of the uniaxial apparent modulus of human trabecular bone using macroscopic mechanical tests and nanoindentation.," J. Biomech., vol. 40, no. 15, pp. 3333-40, Jan. 2007.

[41] G. H. van Lenthe, M. Stauber, and R. Müller, "Specimen-specific beam models for fast and accurate prediction of human trabecular bone mechanical properties.," Bone, vol. 39, no. 6, pp. 1182-9, Dec. 2006.

[42] R. Hambli, "Micro-CT finite element model and experimental validation of trabecular bone damage and fracture.," Bone, vol. 56, no. 2, pp. 363-74, Oct. 2013.

[43] S. E. Basler, T. L. Mueller, D. Christen, a J. Wirth, R. Muller, and G. H. van Lenthe, "Towards validation of computational analyses of peri-implant displacements by means of experimentally obtained displacement maps.," Comput. Methods Biomech. Biomed. Engin., vol. 14, no. 2, pp. 165-74, Feb. 2011. 
Figure legends:

Fig. 1: Peri-implant bone damage was assessed by conducting two sets of $\mu \mathrm{CT}$ scans; once before and once after screw insertion. Image registration was used (step a) to align $\mu C T$ scan 1 with $\mu C T$ scan 2 (step b). Next, both grey scale images were segmented to obtain the bone and screw structures (step c). In the last step (d), the segmented screw from the physical insertion (PI) model was copied and digitally inserted into the segmented image of $\mu \mathrm{CT}$ scan 1 to obtain the digital insertion model; the latter does not contain any peri-implant damage.

Fig. 2: The images on the left and right side show representative examples of a physical insertion (PI) and a digital insertion (DI) model, respectively. A visual comparison between the PI and DI model showed clear evidence for peri-implant bone damage due to screw insertion (representative locations shown with red arrows).

Fig. 3: In an overlay procedure, the digital insertion (DI) and physical insertion (PI) image were combined to produce an overlay image. In the overlay image, two specific voxel-labeling categories were defined: (1) bone present in both images (transparent grey) and (2) bone present in the $\mathrm{PI}$ image only (pink). The bone in category 2 represents bone that has been displaced due to pre-hole drilling and screw insertion remains within close vicinity of the screw. The red arrows point out representative locations.

Fig. 4: The precision of the entire rescanning and registration process was assessed underneath the screw where no architectural changes were expected. More specifically, all bone voxels belonging to category 2 (Fig.3) were quantified in 14 cylindrical volumes of interest (VOI; all $0.3 \mathrm{~mm}$ in height and $5.4 \mathrm{~mm}$ in diameter) underneath the screws and normalized to the bone volume (BV) of the VOI (Fig 4a). Bone damage density was assessed in hollow cylindrical VOls around the screw in a range from 0.0 to $5.4 \mathrm{~mm}$ in steps of $0.3 \mathrm{~mm}$ (Fig $4 \mathrm{~b}$ ) by computing bone damage volume (BDV) normalized to the bone volume $(\mathrm{BDD}=\mathrm{BDV} / \mathrm{BV})$.

Fig. 5: Bone damage of a representative specimen visualized (a) with a side view of the entire bone core and (b) with a virtual mid-section cut along the axial orientation of the screw. The screw was removed to get a clear view on bone damage throughout the entire specimen. Bone damage densities as a function of the radial distance small thread size (STS) screw (c) and large thread size (LTS) screw (d), respectively. Bone damage density was defined as the bone damage volume normalized to bone volume. 
a)

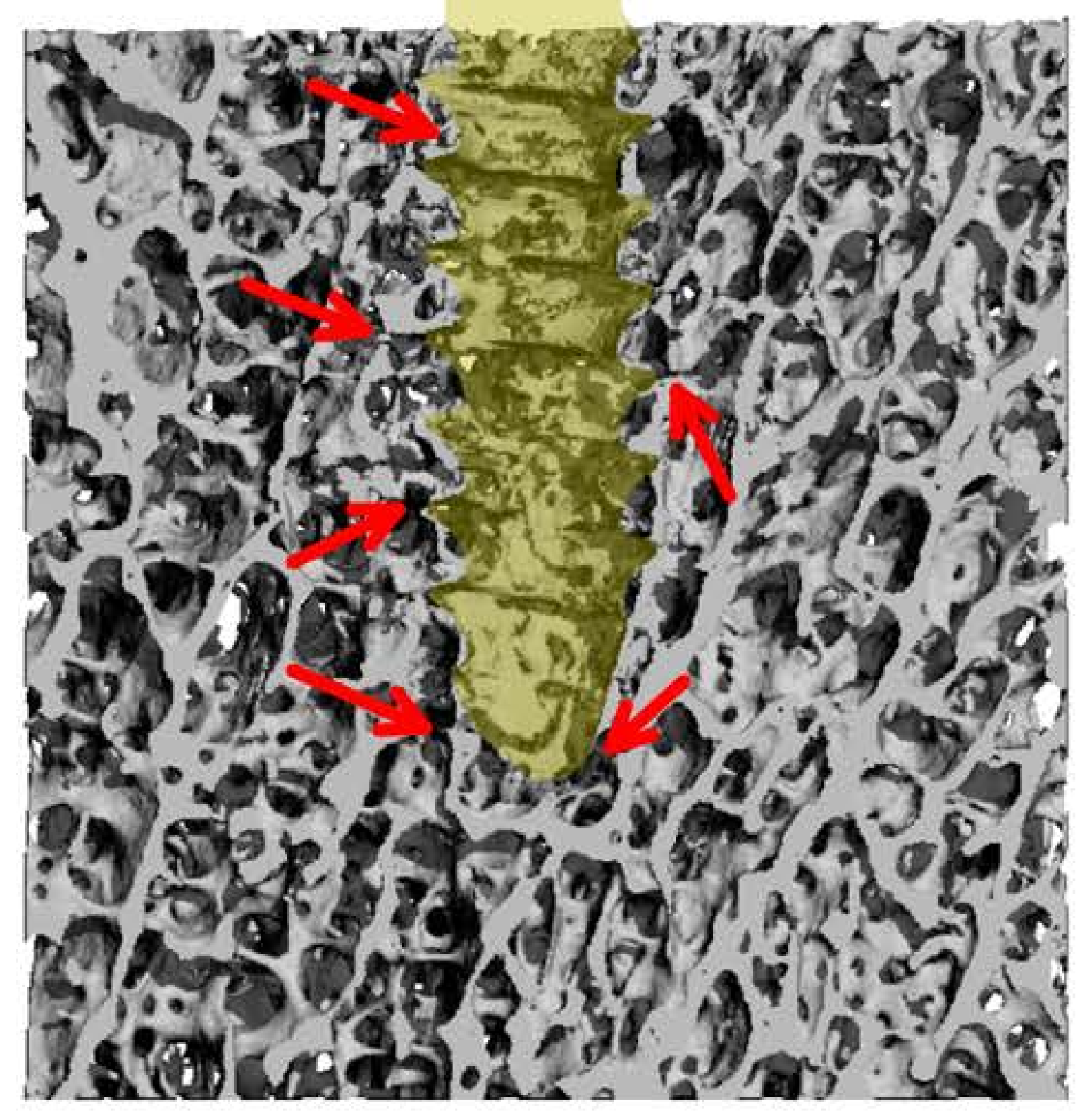

b)

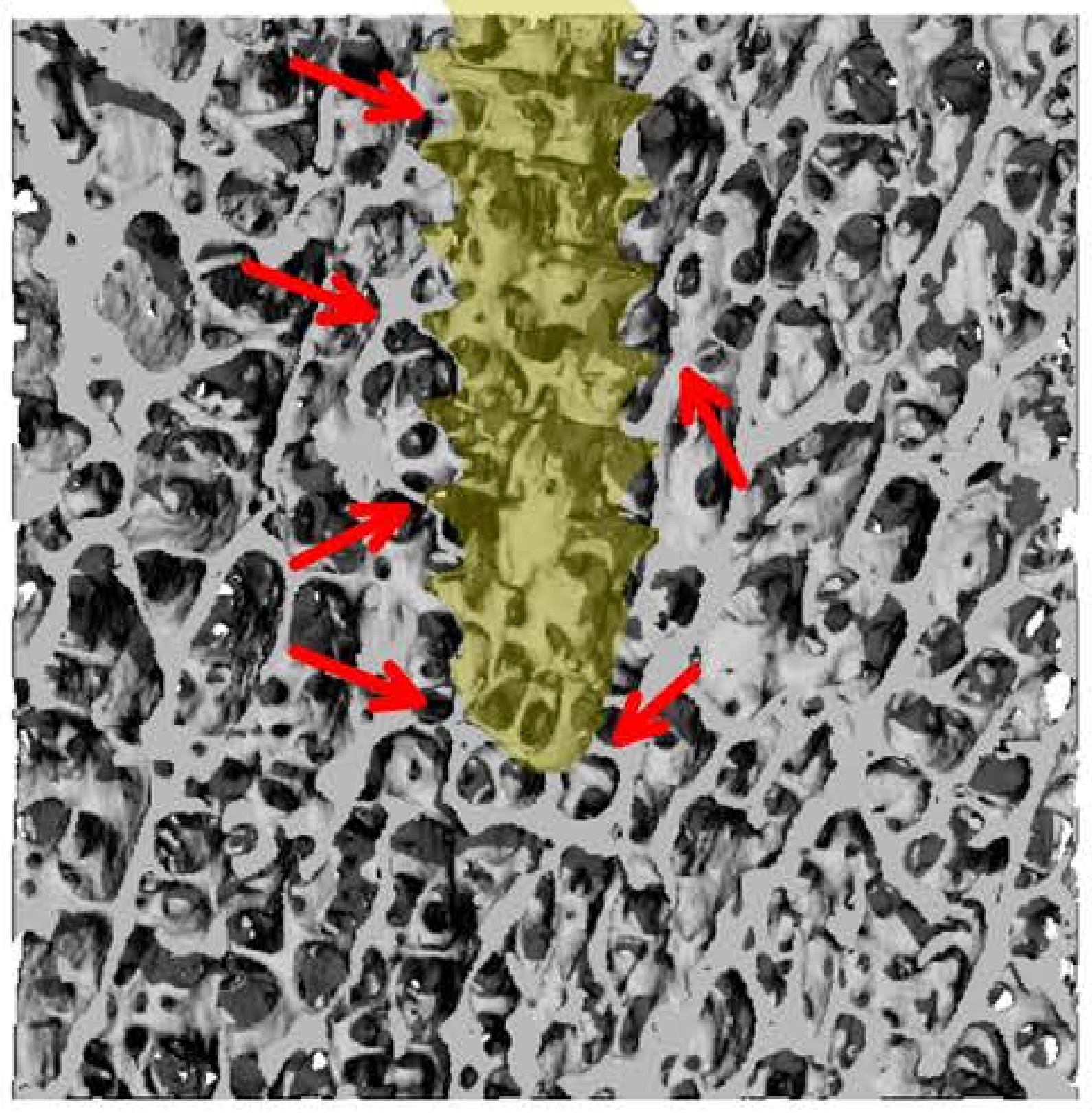

DI model 


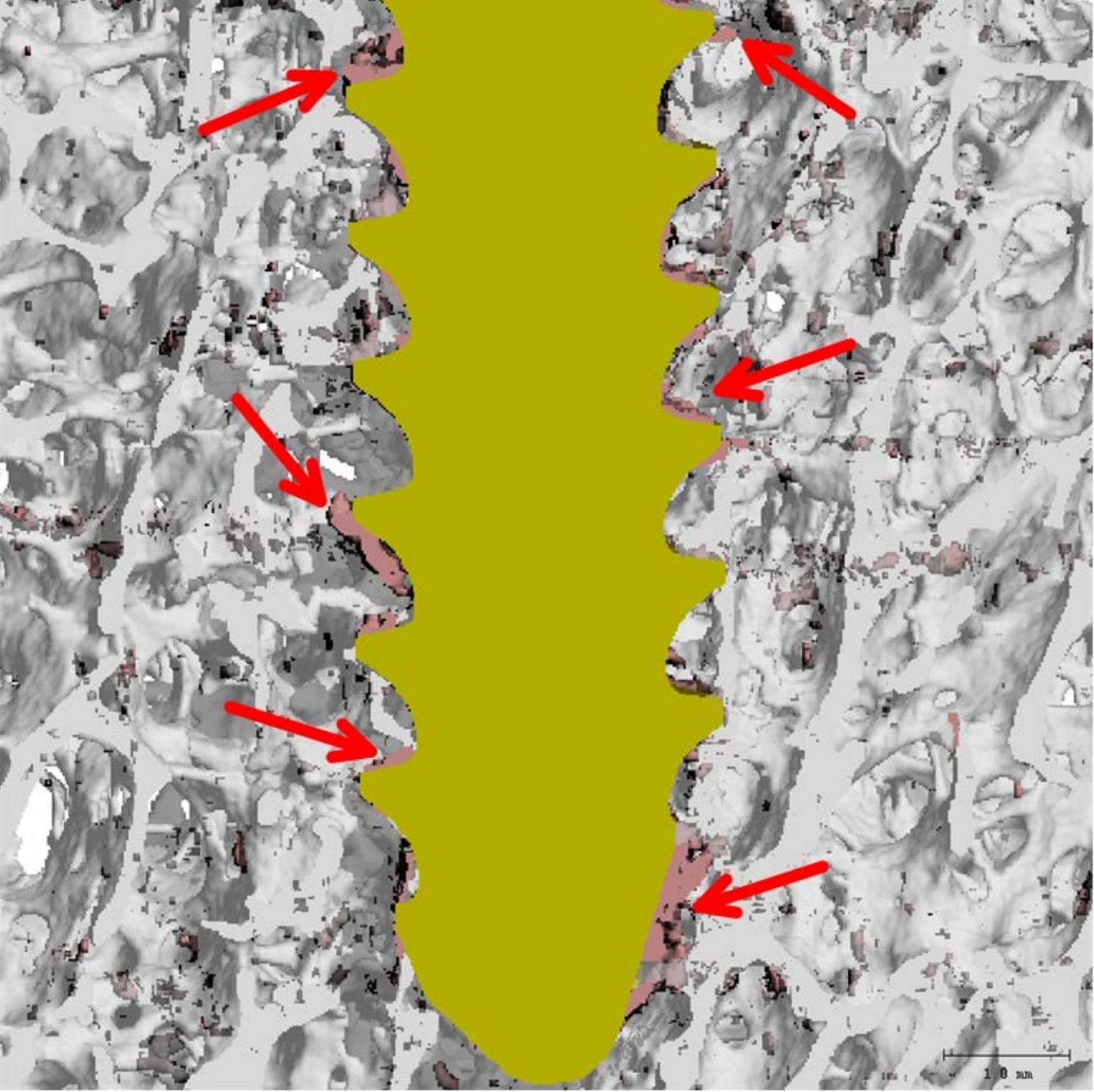


a)

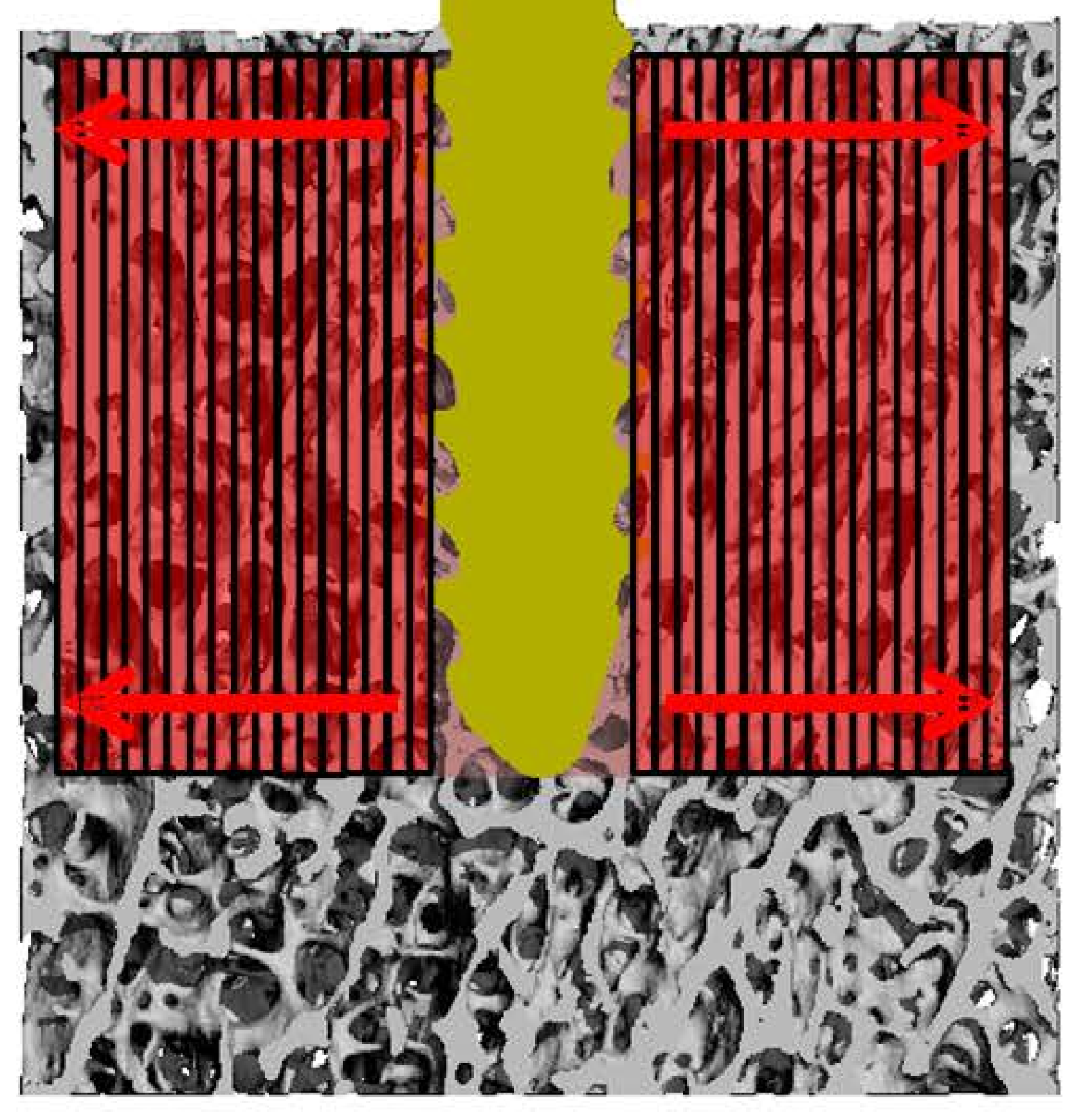

b)

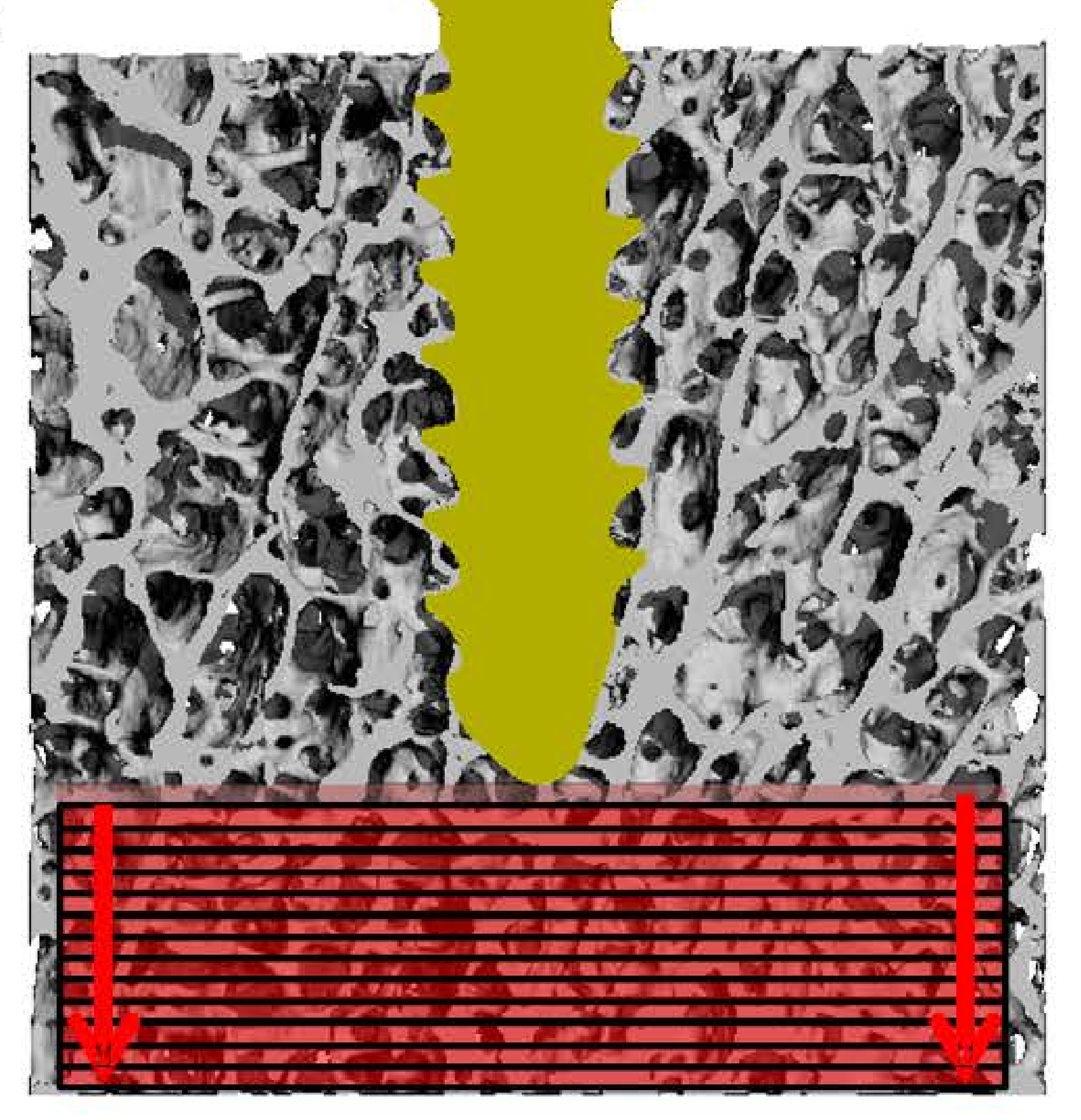


